\title{
A Mathematical Strategy to Relate Fire Safety Evaluation and Fire Safety Policy Formulation for Buildings
}

\author{
H. A. DONEGAN, T. J. SHIELDS, and G. W. SILCOCK
}

University of Ulster

\section{ABSTRACT}

The basis for this study has its origin in the perceived need for the continued development of more rigorous but manageable fire safety evaluation strategies. The schemes which were particularly studied incorporated at least implicitly a form of hierarchical structure with Delphi driven data links utilising a notional form of cross-impact analysis to determine priority rankings for fire safety components. Anomalies were apparent in relation to the underlying philosophy of the procedures adopted in the establishment of the component rankings.

The paper addresses the problem at two levels:

1. The fundamental level - where an axiomatic approach is taken with respect to

- defining a hierarchy in relation to fire safety

- explaining the meaning of interactive importance

- proposing the notion of a partial impact

- defining a total impact

- introducing sequential perturbation.

2. The pragmatic level - where a rigorous numerical approach is taken with respect to

- maintaining the Delphi assessment interval at each stage of the quantification

- stretching the component rankings to enhance the more important components and decrease the significance of the less important components

- weighting consensus data with perturbation noise. 


\section{INTRODUCTION}

It is clear from the literature that fire safety evaluation is in general a very complex issue in the total senario of fire safety. Evaluation generally relies on some form of quantification and quantification relies on a model of the situation. If the situation behaves in a totally irrational manner then coherent and logical models are difficult to produce. However over the years the evolution of prescriptive legislation has to some extent lessened the irrationality factor and in consequence there has been considerable growth in expert opinion. The combination of these two factors enables the Delphi technique [4] to be utilised in providing a quantification basis for mathematical models of that type necessary to study fire safety evaluation.

A typical model which has been researched by the authors in relation to a fire safety points scheme for dwellings is characterised in practical terms by the illustration in Fig 1 .

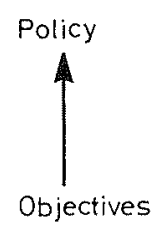

$\uparrow$

Tactics<smiles>CCCC</smiles>

Components
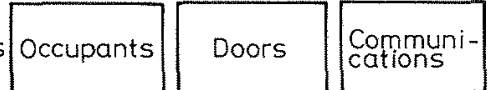

Fire Safety

\section{FIGURE 1 Typical Hierarchy}

The various blocks in the diagram interact with each other from one level to the next and within each level. These interactions beg the question of quantification and measure. The Delphi technique introduces numbers into the system but not without some serious methodological problems. These have been addressed by the authors in [5]. See also [6]. The aim of the model is to relate Components to Policy using basic consensus data at each level. This poses problems with regard to the definition of cross-impacts and their quantification. Typically if a consensus Delphi rating on a scale from 0 to 5 is 4.5 relating for example Travel Distance to Safe Egress, the consequent calculations for Travel Distance to say Life Safety might yield a score of 6.75 outside the $0-5$ range. In this model the $0-5$ bound is maintained. Fundamentally the Components to Policy weighing achieved from this model is, in photographic terms, a freeze frame weighting. 
In other words it is a measure of Policy influence on Components 'now' without any time variable. It is however, possible to take the ranked components and project them forward in time using various time dependent models. Fig 4 illustrates the results of such a projection. on a vector from the dwelling scheme using the Cross Impact Primer due to Kane as described in [4].

\section{THE HIERARCHY}

A hierarchy for the purpose of this paper will be considered as a finite ordered family of collections where the intersection of any two collections is null and there exists at most one collection having a single element set known as a the supreme collection.

In this case the family is ordered quitesimply by the relation "Is at a higher level than". Thus from [2] and omitting collections below the level of the component collection, the supreme and subsequent collections are policy, objectives, tactics and components. Ideally the supreme collection is indexed in such a way that it will cater for a local, national or international policy within any of the following sectors, private, residential, commercial/industrial or community. once the index is chosen this subsequently determines the sets within each collection or level.

Thus:

$\begin{aligned} \text { Policy } & =\{\mathrm{P}\} \\ \text { Objectives } & =\left\{\mathrm{O}_{1}, \mathrm{O}_{2}, \ldots ., \mathrm{O}_{\mathrm{q}}\right\} \\ \text { Tactics } & =\left\{\mathrm{T}_{1}, \mathrm{~T}_{2}, \ldots, \mathrm{T}_{\mathrm{n}}\right\} \\ \text { Components } & =\left\{\mathrm{C}_{1}, \mathrm{C}_{2}, \ldots, \mathrm{C}_{\mathrm{m}}\right\}\end{aligned}$

where $\mathrm{m} \geqq \mathrm{n} \geqq \mathrm{q}>1 ; \mathrm{m}, \mathrm{n}$ and $\mathrm{q}$ are positive integers. No physically related ordering is implied by the integral suffix notation used in the elements of each collection. Furthermore it is assumed than any distinct pair of elements within each collection represent a realistic distinction in terms of what they model. However, once the elements are written down in a sequence then that order is preserved for consistency and vectorial representation.

\section{THE DELPHI WEIGHTING}

The necessary data for use in the hierarchy is derived from a Delphi enquiry see [2] and [5]. After consensus, each element within the collection is represented by means of a vector. Each entry in the vector represents the importance of an interaction with an element from the next higher level collection. For example $C_{i} \backslash \mathrm{T}_{j}$ is the interaction between the ith component and the $j$ th tactic with a corresponding measure of importance given by $t_{i j}$. Thus for all the collections we have the following interactions and their corresponding measures of importance:

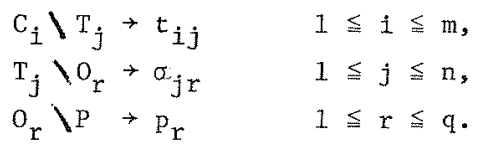


Figure 2 illustrates these measures in terms of the total hierarchy.

Policy (supreme) collection -

Objectives collection-

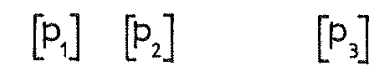

Objectives collection -<smiles></smiles>

$$
\left[\begin{array}{l}
\sigma_{11} \\
\sigma_{12} \\
\sigma_{1 q}
\end{array}\right]
$$$$
\left[\begin{array}{l}
\sigma_{n 1} \\
\sigma_{n 2} \\
\sigma_{n q}
\end{array}\right]
$$

Tactics collection -

$$
\begin{gathered}
\left\{T_{1}\right. \\
{\left[\begin{array}{l}
t_{11} \\
t_{12} \\
t_{1 n}
\end{array}\right] \quad\left[\begin{array}{l}
t_{21} \\
t_{22} \\
t_{2 n}
\end{array}\right]}
\end{gathered}
$$$$
\left[\begin{array}{c}
\sigma_{21} \\
\sigma_{22} \\
\sigma_{2 q}
\end{array}\right]
$$

$\left.T_{n}\right\}$

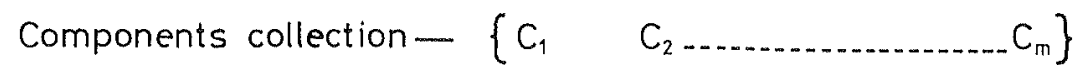

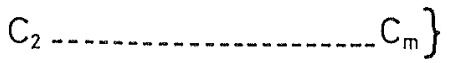

FIGURE 2 Interaction Vectors

\section{IMPACTS}

The aim of the model is to produce an interaction array depicting measures of component importance relative to policy. The following mathematical strategy is fundamental to the derivation of this array.

It is postulated that as a result of cross impacts, the collection of tactics impacts on the collection of components relative to each element in the objectives collection in such a way that the collection of components now assumes new importance relative to the higher objectives level. If now the collection of objectives impacts on the collection of components relative to the singleton policy element the result is a measure of importance on the collection of components relative to the supreme collection.

Each element of the collection of tactics will have a partial impact on each component for every element in the collection of objectives. For example the partial impact of $\mathrm{T}_{j}$ on $\mathrm{C}_{i}$ relative to $\mathrm{O}_{\mathrm{r}}$ may be denoted by:

$\partial I\left[C_{i} / T_{j}\right]_{O_{r}}$ 
This is quantified as the image of a product mapping from

$[0,5] \times[0,5] \rightarrow \mathbb{R}$ (the set of real numbers)

by

$\partial I\left[C_{i} / T_{j}\right]_{O_{r}}=\left(C_{i} \backslash T_{j}\right) \times\left(T_{j} \backslash O_{r}\right)$

which from figure 2 implies

$\partial \mathrm{I}\left[\mathrm{C}_{i} / \mathrm{T}_{j}\right]_{\mathrm{O}_{\mathrm{r}}}=\mathrm{t}_{i j} \sigma_{j \mathrm{r}}$

The total impact of the collection of tactics on $c_{i}$ is defined as the sum of the partial impacts thus:

$$
\begin{aligned}
C_{i}(\text { Tactics }) O_{r} & =\sum_{j=1}^{n} \partial I\left[C_{i} / T_{j}\right]_{o_{r}} \text { for each } r \\
& =\sum_{j=1}^{n} t_{i j} \sigma_{j r} \text { for each } r .
\end{aligned}
$$

From this it is clear that the impacts can be represented as elements of the matrix (C/O) emerging from the product of the components to tactics interaction matrix $(\mathrm{C} / \mathrm{T})$ with the tactics to objectives interaction matrix $(\mathrm{T} / 0)$.

It is now possible to evaluate the new importance weightings of the component collection relative to the objective collection. Since the total impact of the collection of tactics on $C_{1}$ is the sum of $n$ partial impacts for each $r$ see (5) then the average impact density is given by

$\frac{1}{n} \sum_{j=1}^{n} \partial \mathrm{r}\left[\mathrm{C}_{1} / \mathrm{T}_{\mathrm{j}}\right]_{\mathrm{O}_{\mathrm{r}}}$

The new level of importance of $C_{i}$ relative to $o_{r}$ is defined as $C_{i} \backslash O_{r}=\left[\frac{1}{n} \sum_{j=1}^{n} \partial I\left[C_{i} / T_{j}\right]_{O_{r}}\right]^{1 / 2}$

This defines the $m \times q$ components to objectives interaction matrix $(\mathrm{C} / \mathrm{O})^{\prime}$.

In a similar manner the partial impact of each objective on each component relative to $\mathrm{P}$ can be deduced such that:

$$
\begin{aligned}
\partial I\left[C_{i} / O_{r}\right]_{p} & =\left(C_{i} \backslash O_{r}\right) \times\left(O_{r} \backslash P\right) \\
& =\left[\frac{1}{n} \sum_{j=1}^{n} t_{i j} \sigma_{j r}\right]^{\frac{1}{2}} \times p_{r} ; \begin{array}{l}
1 \leqq i \leqq m \\
1 \leqq r \leqq q
\end{array} .
\end{aligned}
$$

Hence the total impact of the collection of objectives on $C_{i}$ is given as:

$C_{i}$ (objectives) $P=\sum_{r=1}^{q} p_{r}\left[\frac{1}{n} \sum_{j=1}^{n} t_{i j} \sigma_{j r}\right]^{\frac{1}{2}}$.

Hence the matrix product $(\mathrm{C} / \mathrm{O})^{\prime} \times(\mathrm{O} / \mathrm{P})$ where $0 / \mathrm{P}$ is the objectives to policy interaction matrix yields the components to policy impact matrix $(\mathrm{C} / \mathrm{P})$. 
The new level of importance of $C_{i}$ relative to $P$ is now given by:

$$
\begin{aligned}
C_{i} \backslash P & =\left[\frac{1}{q} \sum_{r=1}^{q} \partial\left[\left[C_{i} / O_{r}\right]_{p}\right]^{\frac{1}{2}}\right. \\
& =\left[\frac{1}{q} \sum_{r=1}^{q} p_{r}\left[\frac{1}{n} \sum_{j=1}^{n} t_{i j} \sigma_{j r}\right]^{\frac{1}{2}}\right]^{\frac{1}{2}} .
\end{aligned}
$$

This specifies the colum vector $(\mathrm{C} / \mathrm{P})^{\prime}$ i.e. the interaction array of components relative to policy.

\section{NUMERICAL CONSIDERATIONS}

Each entry $x_{i}$ in (C/P)' will lie in the range $0 \leqq x_{i} \leqq 5$, $(I \leqq i \leqq m)$ and $i$ general $x_{i}$ will have a three decimal place format yielding a component priority listing but with a regression toward the mean. In order that the relative percentage weighting of each entry becomes more significant the following transformation is applied to each $\mathrm{x}_{\mathbf{i}}$.

$x_{i} \rightarrow x_{i}^{\prime}=x_{i}-\left(\bar{x}-2 \max \left|\bar{x}-x_{i}\right|\right) ; 1 \leqq i \leqq m$,

where $\bar{x}$ is the geometric mean of all the $x_{i}$. The percentage weightings are then established for each component as ${ }^{1}$ follows:

$x_{i}^{*}=100\left[\left(x_{i}^{*}\right)^{2} / \sum_{1}^{n}\left(x_{i}^{*}\right)^{2}\right]$

\section{SEQUENTIAL PERTURBATIONS}

The model which has been described up to this point ignores the possibility of pairwise set comparisons within each level. These comparisons are identified as levels of importance in square interaction matrices. The interactions are scored as previously on a continuous scale from 0 to 5 relative to each set belonging to the next highex level. Thus for the $m$ Components there are $n$ Pairwise Comparison Interaction (PCI) matrices corresponding to each of the $n$ tactics. Similarly for the $\mathrm{n}$ tactics there are $\mathrm{q}$ PCI matrices and for the q objectives there is one PCI matrix corresponding to the policy $P$.

In view of the fact that many of the matrices will be sparse the technique for evaluating the consequence of their impact is more constrained than the procedure already described. The procedure ill be outlined at the components level. Fundamentally the question which is asked takes the form: "Is there an interaction between $C_{i}$ and $\mathrm{C}_{j}(1 \leqq j \leqq \mathrm{~m})$ relative to the tactic $\mathrm{T}_{\mathrm{k}}$ ?" If there is no interaction the score is zero otherwise $0<$ score $\leqq 5$. The implication of this is that the PCI matrix will always be symmetrical.

Consider the Components to Tactics matrix (C/T) as n-column vectors within the m-dimensional component space. The PCI matrix $\left(\mathrm{C}_{\mathrm{m}}\right)_{\mathrm{T}_{\mathrm{k}}}(1 \leqq \mathrm{k} \leqq \mathrm{n})$, can then perturb the vector $\mathrm{T}_{\mathrm{k}}$. Essentially we are looking at the impact of the collection of components on itself relative to $T_{k}$. However, because of the potential sparcity within $\left(\mathrm{C}_{\mathrm{m}}\right)_{\mathrm{T}_{\mathrm{k}}}$ it is possible that the resulting interaction derived from the 
product $\left(\mathrm{C}_{\mathrm{m}}\right) \mathrm{T}_{\mathrm{k}} \times \mathrm{T}_{\mathrm{k}}$ could be far removed from the consensus status of $\mathrm{T}_{\mathrm{k}}$. This problem is overcome by the following method. In order that the consensus status of $\mathrm{T}_{k}$ is not totally destroyed we consider only the 'notse' associated with the perturbation of $\left(\mathrm{C}_{\mathrm{m}}\right)_{\mathrm{T}}$ on $\mathrm{T}_{\mathrm{k}}$. This is accomplished by entering only zeros in the leading diagonal of $\left(\mathrm{C}_{\mathrm{m}}\right) \mathrm{T}_{\mathrm{k}}$ and this becomes axiomatic for all PCI matrices. Thus:

$\left(\mathrm{C}_{\mathrm{m}}\right)_{\mathrm{T}_{\mathrm{k}}} \times \mathrm{T}_{\mathrm{k}}=\mathrm{T}_{\mathrm{k}}^{\mathrm{N}}$ where

$\mathrm{T}_{\mathrm{k}}^{\mathrm{N}}$ is the 'noise' impact vector associated with $\mathrm{t}_{\mathrm{k}}$. The 'noise' interaction vector is then derived using the already established transformation,

$\left[\frac{1}{m-1} \sum_{j=1}^{m} c_{i j} \cdot t_{j k}\right]^{\frac{1}{2}}=t_{j k}^{\prime}$

where $c_{i j}$ is the ijth entry in $\left(C_{m}\right) T_{k}$. The divisor $(m-1)$ and not $m$ is chosen since for all $i(1 \leqq i \leqq m)$ there is by definition at least one zero in the ith row of $\left(\mathrm{C}_{\mathrm{m}}\right) \mathrm{T}_{\mathrm{k}}$.

The noise element $t_{j k}$ is then combined with $t_{j k}$ for each $i$ by taking their quadratic mean, thus:

$\left[\frac{1}{2}\left(t_{j k}^{2}+t_{j k}^{\prime 2}\right)\right]^{\frac{1}{2}}$.

In this manner the consensus vector is weighted with the 'noise' vector to arrive at the peturbed vector $\mathrm{T}_{\mathrm{k}}^{\mathrm{p}}$.

When this process is repeated for each $T_{k}, 1 \leqq k \leqq n$ we write $(\mathrm{C} / \mathrm{T})^{\mathrm{p}}$ as the perturbed matrix of. (C/T). Similarly we establish $(\mathrm{T} / \mathrm{O})^{\mathrm{p}}$ from $(\mathrm{T} / \mathrm{O})$ and $(\mathrm{O} / \mathrm{P})^{\mathrm{P}}$ from $(\mathrm{O} / \mathrm{P})$. These perturbed matrices are then introduced into the Iinear model and a perturbed vector produced relating Components to Policy.

\section{CONCLUDING REMARKS}

The model described above is an essential element of the total scheme illustrated in fig 3 and described in detail in [2].

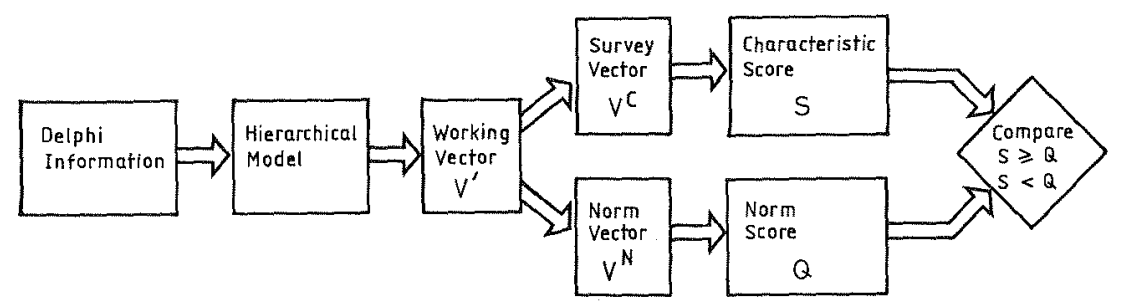

FIGURE 3 Fire Safety Evaluation Scheme 
It is clear that the emergent results are pertinent to a fixed time slot, but it is possible with Delphi influence to introduce a time variable and project the vector forward in real time by some of the established techniques in time series analysis. This projection can be measured relative to the present component trend over say a five year period. Such a prediction could be of value in the development of future fire safety policy and in the preparation of related safety legislation. The authors have used the method of Kane, see [4] to project a typical priority vector and the results are illustrated in $\mathrm{fig} 4$.

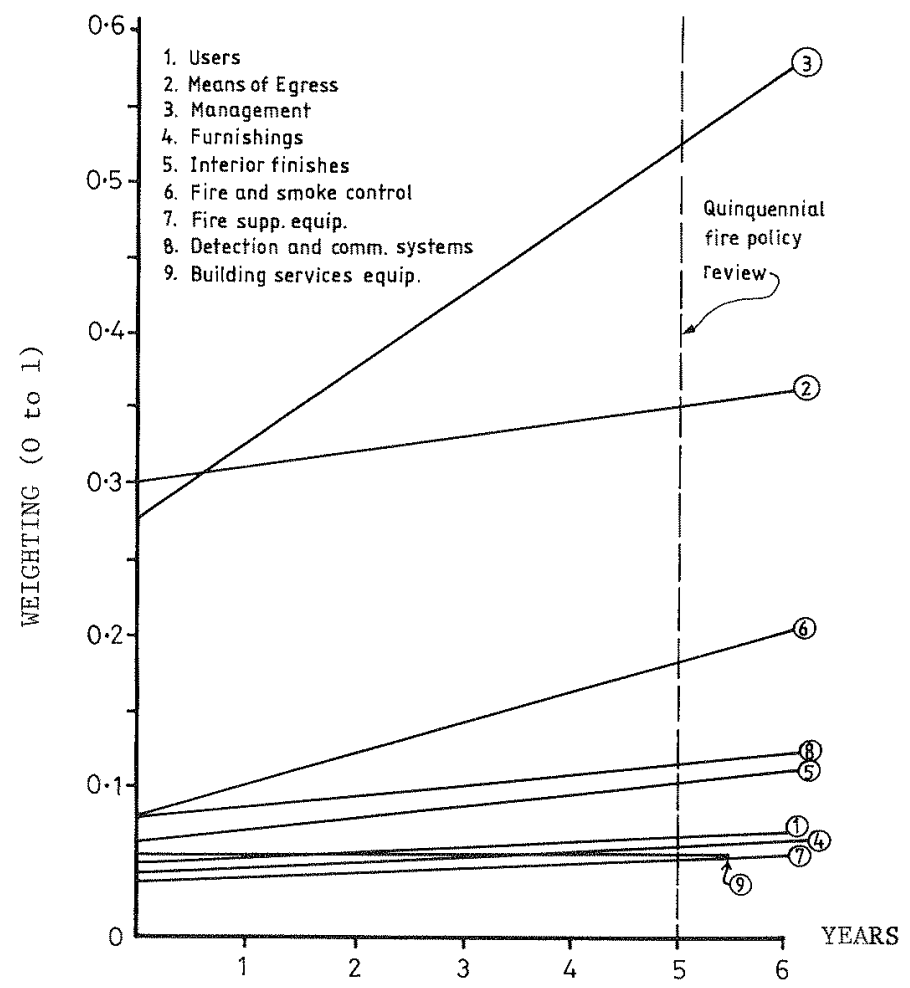

FIGURE 4 Typical Priority Vector Projection

Although future predictions are of considerable interest they lack the strength of internationa? conviction and some form of European Harmonisaiton is to be encouraged under this rationale. See [7]. This would enable a distribution of Hierarchies to be tested and compared internationally.

In preparation for some collaborative European research effort in this field the authors are currently developing software packages which will enable new Delphi data to be processed at a variety of levels. 


\section{REFERENCES}

1. Marchant, E. W., et aI, Fire Safety Evaluation (Points) Scheme for Patient Areas within Hospitals. A report on its origins and development sponsored by DHSS Dept of Fire Safety Engineering, University of Edinburgh 1982.

2. Shields, T. J., Silcock, G. W., Bell, Y., Fire Safety Evaluation of Dwellings, Fire Safety Journal, $10(19 \overline{86}) 29-36$.

3. Shields, T. J., Silcock, G. W., An application of the Hierarchical Approach to Fire Safety, Fire Safety Journal, 11 (1986) 235-242.

4. Linstone, H. A., and Turoff, M., (Eds.), The Delphi Method, Techniques, and Application, Addison Wesley, 1975.

5. Shields, T. J., Silcock, G. W., Donegan, H. A., Methodological Problems associated with the use of the Delphi Technique, Fire Technology, (1987).

6. Helmer, 0., Looking Forward - A guide to Futures Research, Sage Publications, California (1983).

7. Shields, T. J., Silcock, G. W., Donegan, H. A., Devlin, E., Harmonisation and Evaluation of Fire Regulations. Proc. Euro. Fire Reg. Int. Conf. (1987) 17-42. 
